\title{
Prevalence of the potentially inappropriate Kampo medications to be used with caution among elderly patients taking any prescribed Kampo medications at a single centre in Japan: a retrospective cross-sectional study
}

\author{
Junpei Komagamine 1* $^{*}$ and Kazuhiko Hagane ${ }^{2}$
}

\begin{abstract}
Background: Few studies have investigated the prevalence and characteristics of potentially inappropriate Kampo medication use among elderly ambulatory patients taking any prescribed Kampo medications.

Methods: A retrospective cross-sectional study was conducted using electronic medical records. All patients aged 65 years or older who continued visiting internal medicine physicians and were prescribed any Kampo medications from January 2015 to March 2015 were included. The primary outcome was the proportion of patients taking any potentially inappropriate Kampo medications that should be used with caution (hereafter referred to as UWC Kampo medications). The medication appropriateness was evaluated based on the 2015 Japan Geriatrics Society guidelines.
\end{abstract}

Results: Eighty eligible patients were identified. The mean age was 76.7 years, 45 patients (56.2\%) were female, the mean Charlson Comorbidity Index was 1.7, the median number of non-Kampo medications used was 5.0, and the median number of Kampo medications used was 1.0. The proportion of patients taking any UWC Kampo medications was 28.8\% (95\% confidence interval, 18.6 to 38.9\%). Medications containing Glycyrrhizae radix for chronic kidney disease or loop diuretics were the most common UWC Kampo medications. Compared with patients who did not take any UWC Kampo medications, patients who did take such medications used aconite compositions more frequently $(p=0.04)$ and were more likely to have uncontrolled hypertension $(p=0.02)$ and chronic kidney disease $(p<0.001)$. In a multivariable analysis, no predictive factors for the use of UWC Kampo medications were identified.

Conclusions: Approximately one-fourth of the elderly patients taking any prescribed Kampo medications took at least one UWC Kampo medication, although the association between UWC Kampo medications and adverse events remains unclear. When physicians prescribe medications containing Glycyrrhizae radix to elderly patients, chronic kidney disease and the use of loop diuretics should be evaluated.

Keywords: Elderly, Kampo, Japanese herbal medicine, Potentially inappropriate medication

\footnotetext{
* Correspondence: junpei0919@yahoo.co.jp

'Department of Internal Medicine, National Hospital Organization Tochigi

Medical Center, 1-10-37, Nakatomatsuri, Utsunomiya, Tochigi 3208580, Japan

Full list of author information is available at the end of the article
}

(c) The Author(s). 2018 Open Access This article is distributed under the terms of the Creative Commons Attribution 4.0 International License (http://creativecommons.org/licenses/by/4.0/), which permits unrestricted use, distribution, and reproduction in any medium, provided you give appropriate credit to the original author(s) and the source, provide a link to the Creative Commons license, and indicate if changes were made. The Creative Commons Public Domain Dedication waiver (http://creativecommons.org/publicdomain/zero/1.0/) applies to the data made available in this article, unless otherwise stated. 


\section{Background}

Kampo medicine is a form of traditional Japanese herbal medicine. It was introduced to Japan from China 1500 years ago and was developed into a unique form of Japanese medicine over a long period in Japanese practice and culture [1-3]. Therefore, Kampo medicine is different from traditional Chinese and Korean medicines [3]. In Japan, Kampo medicines are integrated into the national health care system; 148 Kampo extract formulations and 187 types of crude drugs are approved by the Ministry of Health, Labour and Welfare and are used under the national health insurance programme [4, 5]. Kampo medications are available over the counter (OTC) and are the most frequently used complementary and alternative medicine therapies in Japan according to surveys of Japanese physicians [6-9].

Despite the common prescription of Kampo medications by Japanese physicians, there are no standard education programmes in medical schools and few postgraduate education programmes on Kampo medicines in Japan $[3,10,11]$. Given that a recent Japanese study reported some concerns regarding patient safety in relation to Kampo medications [4], it is important to ascertain the safety of the Kampo medications prescribed by physicians. Furthermore, given that adverse drug events are common in elderly patients due to multi-morbidity and polypharmacy [12-15], the appropriate use of Kampo medications by elderly patients is particularly important. Nonetheless, to our knowledge, no studies have investigated the appropriateness of the Kampo medications prescribed by physicians as part of typical care in Japan. Thus, our aim was to determine the prevalence of and the factors associated with the use of potentially inappropriate Kampo medications among elderly patients who take any prescribed Kampo medication.

\section{Methods}

\section{Study design and location}

The National Hospital Organization Tochigi Medical Center is a 350-bed community hospital in the Tochigi Prefecture of Japan. The centre is one of the two largest acute care hospitals in the area and serves approximately 0.5 million individuals. At our hospital, the internists mainly practise Western medicine but occasionally use Kampo medicine, depending on the patients' and physicians' preferences. No physicians receive formal training in Kampo medicine after graduation from a medical university. The aim of this study was to determine the prevalence and characteristics of elderly patients who were prescribed potentially inappropriate Kampo medications. A retrospective, single-centre, cross-sectional study was conducted using the electronic medical records of National Hospital Organization Tochigi Medical Center. The primary outcome was the proportion of patients who were prescribed at least one potentially inappropriate Kampo medication to be used with caution (hereafter denoted as UWC Kampo medications) among elderly ambulatory patients who were regularly prescribed any Kampo medications. We also evaluated the factors associated with the UWC Kampo medications. This study was approved by the Medical Ethical Committee of National Hospital Organization Tochigi Medical Center (No. 29-10) and was conducted in accordance with the Declaration of Helsinki. The need for individual informed consent was formally waived by the Medical Ethical Committee of National Hospital Organization Tochigi Medical Center because the study used data collected from medical records and the patients were not contacted.

\section{Participants and inclusion criteria}

All consecutive ambulatory patients aged 65 years or older who had appointments with internal medicine physicians from January 1, 2015, to March 31, 2015, were retrospectively screened. This study included only patients who had 3 or more visits to internal medicine physicians within a year before the index visit and who were prescribed at least one Kampo medication on two or more consecutive visits. Over-the-counter Kampo medications were excluded. Patients with missing data regarding medications prescribed at other hospitals were excluded. During the study period, 1035 ambulatory patients aged 65 years or older were identified. Nine hundred fifty-five patients were excluded for a variety of reasons (895 for a lack of regular use of Kampo medications and 60 for missing data on medications prescribed at other hospitals). Thus, a total of 80 patients were included in the final analysis.

\section{Data collection}

The data were collected using the electronic medical records of National Hospital Organization Tochigi Medical Center. All available electronic medical records from September 2014 to the time of the index visit were reviewed. Information regarding age, gender, past medical history, the Charlson Comorbidity Index (CCI) [16], office blood pressure, non-Kampo medications, and Kampo medications was retrieved. All prescriptions from our hospital were covered by the hospital database, but prescriptions from other hospitals were not covered. Therefore, prescriptions from other hospitals were identified based on medical record documentation by physicians in our hospital. The non-Kampo medications included oral medications, inhalers, injections, and as-needed medications. However, eye drops, intranasal infusers, OTC drugs, and topical medications were excluded, along with medications that were indicated for apparent transient disease and those that 
were not prescribed on 2 or more consecutive visits. For example, antitussive medications for the common cold and antibiotic agents for pharyngitis were not included. The numbers of non-Kampo and Kampo medications were counted separately.

\section{Outcome measures}

The primary outcome was the proportion of elderly patients who took any UWC Kampo medications among those who took at least one prescribed Kampo medication. We defined UWC Kampo medications based on the 2015 traditional Chinese medicine Japan Geriatrics Society guidelines [17]. The following five criteria were used: (1) aconite composition use among patients with uncontrolled hypertension or tachyarrhythmia; (2) Glycyrrhizae radix composition use among patients with chronic kidney disease (CKD) or loop diuretic use; (3) ephedra composition use among patients with uncontrolled hypertension, ischaemic heart disease, or dysuria; (4) Scutellaria baicalensis composition use among patients with interferon use or liver cirrhosis; and (5) longterm use of gardenia composition (more than a few years). The representative Kampo medications that included these components and were identified in this study are shown in Table 1. Uncontrolled hypertension was defined as office systolic blood pressure $\geq 140 \mathrm{mmHg}$ and/or diastolic blood pressure $\geq 90 \mathrm{mmHg}$ [18-20]. If office blood pressure was not documented in medical records, hypertension was judged to be uncontrolled for patients whose antihypertensive medications were increased over three or more consecutive physician visits. Otherwise, hypertension was judged to be controlled. Tachyarrhythmia was defined as any arrhythmia that was controlled by antiarrhythmic medications. Only patients who were regularly prescribed medications for urinary problems were considered to have dysuria [21-23].

Table 1 Kampo medications containing aconite, Glycyrrhizae radix, ephedra, Scutellaria baicalensis or gardenia that were prescribed for 80 ambulatory elderly patients

\begin{tabular}{ll}
\hline Ingredient & Kampo medications \\
\hline Aconite & Hachimijiogan, Goshajinkigan \\
Glycyrrhizae radix & Yokukansan, Shakuyakukanzoto, \\
& Bakumondoto, Kakkonto, Rikkunshito, \\
& Keishikashakuyakuto, Shakanzoto, \\
& Shoseiryuto, Ryokeijutsukanto, Junchoto, \\
& Hochuekkito, Kakkontokasenkyushini, \\
& Hangeshashinto, Saikokeishikankyoto, \\
& Shokenchuto, Kososan, Nijutsuto, Saireito, \\
& Bouiogito \\
Ephedra & Kakkonto, Shoseiryuto, Kakkontokasenkyushini \\
Scutellaria baicalensis & Junchoto, Hangeshashinto, Saikokeishikankyoto, \\
& Nijutsuto, Unseiin, Saireito \\
Gardenia & Unseiin \\
\hline
\end{tabular}

Long-term use of gardenia composition was defined as the use of gardenia for more than 3 years because the term "a few years", which is used in the 2015 Japan Geriatrics Society guidelines [17], is ambiguous.

\section{Statistical analysis}

The sample size was determined based on the following information. The interval between consecutive regular visits for most of the ambulatory patients at our hospital was a few months. Therefore, the patients who attended at least one physician visit from January 1, 2015, to March 31, 2015, were screened. The characteristics of the included patients were described using descriptive statistics. The primary outcome was the proportion of patients who were prescribed any UWC Kampo medication. The 95\% confidence interval (CI) was calculated for this outcome. To compare the patient characteristics of patients who were and were not prescribed any UWC Kampo medications, Fisher's exact tests were used for categorical variables, and Student's t-tests were used for continuous variables. To identify the determinants of the prescription of UWC Kampo medications, multivariable analysis using binary logistic regression was also conducted. The following variables were included in the logistic regression model: age, gender, $\mathrm{CCI}$, number of non-Kampo medications used, and the use of multiple Kampo medications. These analyses were conducted using IBM SPSS Statistics Base version 21.0 (IBM Corporation, Nihonbashi, Tokyo, Japan) or Excel statistical software package version 2.11 (Bellcurve for Excel; Social Survey Research Information Co., Ltd., Tokyo, Japan). The threshold for statistical significance was $p<0.05$.

\section{Results}

The characteristics of the included patients are presented in Table 2. Among the 80 patients, the mean age was 76.7 years, $45(56.2 \%)$ were female, the mean CCI was 1.7 , the median number of non-Kampo medications used was 5.0, and the median number of Kampo medications used was 1.0. The numbers of patients who took prescribed Kampo medications containing aconite, Glycyrrhizae radix, ephedra, Scutellaria baicalensis, and gardenia were 8 (10.0\%), 57 (71.3\%), 10 (12.5\%), 6 (7.5\%), and $1(1.3 \%)$, respectively.

The proportion of patients who were prescribed any UWC Kampo medications was $28.8 \%$ (95\% CI, 18.6 to 38.9\%; Table 3). Compared with patients who did not take any UWC Kampo medications, patients who did take such medications used aconite composition more frequently $(p=0.04)$ and were more likely to have uncontrolled hypertension $(p=0.02)$ and CKD $(p<0.001)$. The proportion of female gender and use of multiple Kampo medications tended to be higher in patients who took any UWC Kampo medications than in patients 
Table 2 Characteristics of the elderly ambulatory patients and the comparison of patients who were prescribed any potentially inappropriate Kampo medications to be used with caution and those who were not

\begin{tabular}{|c|c|c|c|c|}
\hline & & Prescription & ropriate Kampo medications ${ }^{a}$ & $p$-value \\
\hline & $\begin{array}{l}\text { Total } \\
n=80\end{array}$ & $\begin{array}{l}\text { Yes } \\
n=23\end{array}$ & $\begin{array}{l}\text { No } \\
n=57\end{array}$ & \\
\hline Age, mean $\pm S D$ & $76.7 \pm 8.1$ & $78.1 \pm 8.3$ & $76.2 \pm 8.0$ & 0.34 \\
\hline Female, $n(\%)$ & $45(56.2)$ & $17(73.9)$ & $28(49.1)$ & 0.05 \\
\hline Charlson Comorbidity Index, mean \pm SD & $1.7 \pm 1.8$ & $1.8 \pm 2.0$ & $1.6 \pm 1.8$ & 0.62 \\
\hline Non-Kampo medications ${ }^{c}$ & & & & \\
\hline Total number & & & & \\
\hline Median (IQR) & $5.0(3.0-7.3)$ & $6.0(4.0-8.5)$ & $5.0(3.0-7.0)$ & 0.80 \\
\hline Mean \pm SD & $5.6 \pm 3.0$ & $6.4 \pm 3.1$ & $5.3 \pm 3.0$ & 0.16 \\
\hline Five or more medications, $n(\%)$ & $46(57.5)$ & $14(60.9)$ & $32(56.1)$ & 0.80 \\
\hline Kampo medications & & & & \\
\hline Total number & & & & \\
\hline Median (IQR) & $1.0(1.0-1.0)$ & $1.0(1.0-2.0)$ & $1.0(1.0-1.0)$ & NA \\
\hline Mean \pm SD & $1.2 \pm 0.4$ & $1.3 \pm 0.5$ & $1.1 \pm 0.3$ & 0.05 \\
\hline Two or more medications, $n$ (\%) & $14(17.5)$ & $7(30.4)$ & $7(12.3)$ & 0.10 \\
\hline Aconite composition, $n$ (\%) & $8(10.0)$ & $5(21.7)$ & $3(5.3)$ & 0.04 \\
\hline Glycyrrhizae radix composition, $n(\%)$ & $57(71.3)$ & $19(82.6)$ & $38(66.7)$ & 0.18 \\
\hline Ephedra composition, $n$ (\%) & $10(12.5)$ & $3(13.0)$ & $7(12.3)$ & 1.00 \\
\hline Scutellaria baicalensis composition, $n(\%)$ & $6(7.5)$ & $2(8.7)$ & $4(7.0)$ & 1.00 \\
\hline Gardenia composition, n (\%) & $1(1.3)$ & $1(4.4)$ & $0(0.0)$ & 0.28 \\
\hline Specific medications, $n$ (\%) & & & & \\
\hline Three or more antihypertensive drugs & $14(17.5)$ & $7(30.4)$ & $7(12.3)$ & 0.10 \\
\hline Loop diuretics & $6(7.5)$ & $1(4.4)$ & $5(8.8)$ & 0.67 \\
\hline Dysuria medications & $9(11.3)$ & $4(17.4)$ & $5(8.8)$ & 0.43 \\
\hline Interferon & $0(0.0)$ & $0(0.0)$ & $0(0.0)$ & NA \\
\hline Uncontrolled hypertension ${ }^{d}, n(\%)$ & $20(25.0)$ & $10(43.5)$ & $10(17.5)$ & 0.02 \\
\hline Past medical history, $n$ (\%) & & & & \\
\hline Tachyarrhythmia $^{e}$ & $3(3.8)$ & $0(0.0)$ & $3(5.3)$ & 0.55 \\
\hline Dementia & $13(16.3)$ & $4(17.4)$ & $9(15.8)$ & 1.00 \\
\hline Ischaemic heart disease & $7(8.8)$ & $1(4.4)$ & $6(10.5)$ & 0.45 \\
\hline Chronic kidney disease & $25(31.3)$ & 18 (78.3) & $7(12.3)$ & $<0.001$ \\
\hline Liver cirrhosis & $4(5.0)$ & $2(8.7)$ & $2(3.5)$ & 0.57 \\
\hline Hypertension & $50(62.5)$ & $17(73.9)$ & $33(57.9)$ & 0.21 \\
\hline
\end{tabular}

${ }^{a}$ Defined based on the 2015 Japan Geriatrics Society guidelines

${ }^{b}$ Fisher's exact tests or Student's t-tests were used for comparisons between patients who were and were not prescribed any potentially inappropriate Kampo medications to be used with caution. The threshold for statistical significance was set at $p<0.05$

'Excluding Kampo medications

dUncontrolled hypertension was defined as office systolic blood pressure $\geq 140 \mathrm{mmHg}$ and/or diastolic blood pressure $\geq 90 \mathrm{mmHg}$

'Tachyarrhythmia was defined as any arrhythmia that was controlled with antiarrhythmic medication

who did not, although these associations were not statistically significant. Among the UWC Kampo medications, medications containing Glycyrrhizae radix prescribed for patients with CKD or loop diuretic users were the most commonly used ( $n=16,20.0 \%$ ), followed by those containing aconite prescribed for patients with uncontrolled hypertension or tachyarrhythmia $(n=5,6.3 \%)$.
Table 4 presents a summary of the results of the multivariable analysis using logistic regression to identify predictors of the use of UWC Kampo medications. Among the five included variables (i.e., age, gender, CCI, number of non-Kampo medications, and multiple Kampo medications), no independent predictive factors for the use of UWC Kampo medications were identified. 
Table 3 Prevalence of the use potentially inappropriate Kampo medications to be used with caution ${ }^{a}$ in elderly patients taking any prescribed Kampo medications

\begin{tabular}{|c|c|}
\hline Characteristic & $\begin{array}{l}\text { Total } \\
n=80\end{array}$ \\
\hline $\begin{array}{l}\text { Any potentially inappropriate Kampo medications } \\
\text { to be used with caution, } n(\%)\end{array}$ & $23(28.8)$ \\
\hline $\begin{array}{l}\text { Aconite for uncontrolled hypertension }{ }^{\mathrm{b}} \text { or } \\
\text { tachyarrhythmiac }{ }^{c} \text { patients, } n(\%)\end{array}$ & $5(6.3)$ \\
\hline $\begin{array}{l}\text { Glycyrrhizae radix for CKD patients or loop } \\
\text { diuretic users, } n(\%)\end{array}$ & $16(20.0)$ \\
\hline $\begin{array}{l}\text { Ephedra for uncontrolled hypertension }{ }^{\mathrm{b}} \text {, ischaemic } \\
\text { heart disease, or dysuria patients, } n(\%)\end{array}$ & $1(1.3)$ \\
\hline $\begin{array}{l}\text { Scutellaria baicalensis for interferon users or } \\
\text { liver cirrhosis patients, } n(\%)\end{array}$ & $0(0.0)$ \\
\hline Long-term use ${ }^{\mathrm{d}}$ of gardenia, $n(\%)$ & $1(1.3)$ \\
\hline
\end{tabular}

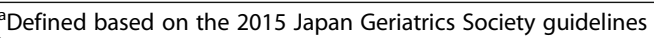

buncontrolled hypertension was defined as office systolic blood pressure $\geq$

$140 \mathrm{mmHg}$ and/or diastolic blood pressure $\geq 90 \mathrm{mmHg}$

'Tachyarrhythmia was defined as any arrhythmia that was controlled with antiarrhythmic medications

dLong-term use was defined as use for more than 3 years

\section{Discussion}

This study found that approximately one-fourth of elderly patients taking prescribed Kampo medications took UWC Kampo medications. Most of the UWC Kampo medications were medications containing Glycyrrhizae radix prescribed for CKD patients or loop diuretic users and medications containing aconite prescribed for patients with uncontrolled hypertension or tachyarrhythmia. Compared with patients who did not take any UWC Kampo medications, those who did used aconite composition more frequently $(p=0.04)$ and were more likely to have uncontrolled hypertension $(p=0.02)$ and CKD $(p<0.001)$.

To our knowledge, the present study is the first to evaluate the appropriateness of Kampo medications prescribed by physicians for elderly patients in Japan. Because no data have been published regarding the appropriateness of Kampo medications prescribed by physicians, it is unknown whether the present results are consistent with data from other hospitals in Japan. However, the prevalence of the use of UWC Kampo medications in this study is similar to the reported prevalences of potential adverse herbdrug interactions among patients using herbal medicines in previous studies [24-27]. Furthermore, the prevalence of the use of potentially inappropriate Western medications among community-dwelling elderly patients ranges from 20 to $40 \%[28,29]$. Therefore, the prevalence of the use of UWC Kampo medications observed in this study might reflect the true prevalence of their use among elderly patients in Japan. Considering the common use of prescribed Kampo medications in Japan [6-9, 30-33], it is surprising that few studies have evaluated the appropriateness of Kampo medication use. Further studies are warranted to determine the prevalence of the use of UWC Kampo medications among elderly patients at other hospitals and in other settings in Japan, and efforts are needed to improve the appropriateness of Kampo medication use among elderly patients. In this study, most of the UWC Kampo medications were medications containing Glycyrrhizae radix prescribed for CKD patients or loop diuretic users and medications containing aconite prescribed for patients with uncontrolled hypertension or tachyarrhythmia. These findings imply that physicians should be cautious when they use Kampo medications containing Glycyrrhizae radix or aconite for elderly patients.

To evaluate the appropriateness of Kampo medication use, we used the definitions of UWC Kampo medications based on the 2015 Japan Geriatrics Society guidelines on traditional Chinese medicine [17]. The three definitions of UWC Kampo medications regarding aconite, Glycyrrhizae radix, and ephedra compositions correspond to contraindications or precautions based on another authoritative resource [34] and review [35]. However, all five definitions applied in this study are based on low-quality studies, such as a case reports and case series [17, 36], because highquality clinical data regarding the safety of herbal medicines are lacking $[35,36]$. Therefore, it is unclear whether UWC Kampo medications are truly associated with worse patient outcomes. Further studies are needed to evaluate

Table 4 Summary results of multivariable logistic regression analyses of predictive factors of the use of potentially inappropriate Kampo medications to be used with caution ${ }^{\text {a }}$

\begin{tabular}{|c|c|c|c|c|}
\hline \multirow[t]{2}{*}{ Variable } & \multicolumn{4}{|c|}{ Odds ratio ( $95 \%$ confidence interval) } \\
\hline & Unadjusted & $p$-value ${ }^{b}$ & Adjusted $^{c}$ & $p$-value ${ }^{b}$ \\
\hline Age & $1.03(0.97-1.09)$ & 0.34 & $1.02(0.96-1.09)$ & 0.50 \\
\hline Female & $2.93(1.01-8.52)$ & 0.047 & $2.95(0.95-9.13)$ & 0.06 \\
\hline Charlson Comorbidity Index & $1.07(0.83-1.38)$ & 0.61 & $1.10(0.82-1.48)$ & 0.52 \\
\hline Number of non-Kampo medications ${ }^{d}$ & $1.12(0.96-1.32)$ & 0.16 & $1.09(0.92-1.30)$ & 0.98 \\
\hline Multiple Kampo medications & $3.13(0.95-10.27)$ & 0.06 & $3.47(0.98-12.26)$ & 0.05 \\
\hline
\end{tabular}

${ }^{\mathrm{a}}$ Defined based on the 2015 Japan Geriatrics Society guidelines

${ }^{\mathrm{b}}$ Statistical significance was evaluated at $p<0.05$

'The following variables were adjusted: age, gender, $\mathrm{CCl}$, number of non-Kampo medications, and multiple Kampo medications

dExcluding Kampo medications 
whether UWC Kampo medications increase the risk of adverse events.

In the multivariable analysis of this study, the use of multiple Kampo medications was not significantly associated with any use of UWC Kampo medications. However, given that both polypharmacy and the number of medications are strong independent risk factors for the use of potentially inappropriate medications (PIMs) among elderly patients in studies of Western medications [28, 37], and multiple Kampo medications should be used with caution. Considering the limitations of this study, e.g., the small sample size and single-centre design, further studies are warranted to evaluate this association between the use of multiple Kampo medications and the use of UWC Kampo medications.

\section{Limitations}

Our results should be interpreted in the context of several limitations. First, the study used a retrospective design. Second, the information on prescriptions from other hospitals might be inaccurate because prescriptions from other hospitals were determined according to the documentation of such prescriptions in patient medical records by physicians at our hospital. Third, the indications for Kampo medications were not evaluated. Therefore, the intended uses of these medications were unclear. Fourth, the use of OTC Kampo medications was not investigated. Furthermore, the transient use of Kampo medications was not considered this study. Fifth, adherence to medication regimens was not assessed. Sixth, we did not evaluate the severity of comorbidities, which might affect the risk of the use of UWC Kampo medications. Seventh, this study was limited to a single centre and included a small sample size. Therefore, these findings should be confirmed by multicentre or populationlevel studies in the future.

\section{Conclusions}

Approximately one-fourth of the elderly patients who took prescribed Kampo medications also took UWC Kampo medications. Medications containing Glycyrrhizae radix prescribed to patients with CKD or loop diuretic users were the most common UWC Kampo medications. Before medications containing Glycyrrhizae radix are prescribed for elderly patients, CKD and loop diuretic use should be checked. These findings need to be confirmed in other settings in Japan.

\section{Abbreviations}

CCl: Charlson Comorbidity Index; Cl: Confidence interval; CKD: Chronic kidney disease; IQR: Interquartile range; NA: Not applicable; OR: Odds ratio; OTC: Over the counter; PIM: Potentially inappropriate medication; SD: Standard deviation; UWC Kampo medication: Potentially inappropriate Kampo medication to be used with caution

\section{Acknowledgements}

We thank the reviewer from BMC Complementary and Alternative Medicine for the help with improving the quality of the manuscript.

\section{Availability of data and materials}

Data sharing is not applicable because we did not receive informed consent for data sharing from the participants.

\section{Authors' contributions}

JK designed the study, collected the data, contributed to data analysis and contributed to writing the manuscript. $\mathrm{KH}$ contributed to the data analysis and to writing the manuscript. Both authors have read and approved the final manuscript.

\section{Ethics approval and consent to participate}

This study was approved by the Medical Ethical Committee of National Hospital Organization Tochigi Medical Center (No. 29-10) and was conducted in accordance with the Declaration of Helsinki. This study was also conducted in accordance with the Ethical Guidelines for

Epidemiological Research in Japan. The need for individual informed consent was formally waived by the Medical Ethical Committee of National Hospital Organization Tochigi Medical Center because the data were collected from medical records and the patients were not contacted. However, per the Japanese Ethical Guidelines, we displayed a poster in the waiting room of the hospital that provided information on the collection and use of data for this study and the protection of personal information.

\section{Competing interests}

The authors declare that they have no competing interests.

\section{Publisher's Note}

Springer Nature remains neutral with regard to jurisdictional claims in published maps and institutional affiliations.

\section{Author details}

'Department of Internal Medicine, National Hospital Organization Tochigi Medical Center, 1-10-37, Nakatomatsuri, Utsunomiya, Tochigi 3208580, Japan. ${ }^{2}$ Department of Paediatric Surgery, National Hospital Organization Tochigi Medical Center, 1-10-37, Nakatomatsuri, Utsunomiya, Tochigi 3208580, Japan.

Received: 22 January 2018 Accepted: 2 May 2018

Published online: 11 May 2018

\section{References}

1. Motoo Y, Seki T, Tsutani K. Traditional Japanese medicine, Kampo: its history and current status. Chin J Integr Med. 2011;17:85-7. https://doi.org/10.1007/ s11655-011-0653-y.

2. Watanabe K, Matsuura K, Gao P, Hottenbacher L, Tokunaga H, Nishimura K, et al. Traditional Japanese Kampo medicine: clinical research between modernity and traditional medicine-the state of research and methodological suggestions for the future. Evid Based Complement Alternat Med. 2011;2011:513842. https://doi.org/10.1093/ecam/neq067.

3. Arai M, Nakada Y, Izumi SI. The education of traditional Japanese (Kampo) medicine: surveys of training hospitals and residents. BMC Complement Altern Med. 2017;17:134. https://doi.org/10.1186/s12906-017-1634-2.

4. Shimada Y, Fujimoto M, Nogami T, Watari H, Kitahara H, Misawa H, et al. Patient safety incident reports related to traditional Japanese Kampo medicines: medication errors and adverse drug events in a university hospital for a ten-year period. BMC Complement Altern Med. 2017;17:547. https://doi.org/10.1186/s12906-017-2051-2.

5. Japanese Ministry of Health, Labour and Welfare. List of the National Health Insurance drugs (Yakka kijun shusai hinmoku risuto) [Japanese Ministry of Health, Labour and Welfare] (in Japanese). 2017. http://www.mhlw.go.jp/ topics/2017/10/dl/tp20171001-01_1.pdf. Accessed 22 Nov 2017.

6. Imanishi J, Watanabe S, Satoh M, Ozasa K. Japanese doctors' attitudes to complementary medicine. Lancet. 1999;354:1735-6. https:/doi.org/10.1016/ S0140-6736(05)76729-5.

7. Watanabe S, Imanishi J, Satoh M, Ozasa K. Unique place of Kampo (Japanese traditional medicine) in complementary and alternative medicine: a survey of doctors belonging to the regional medical association in Japan. Tohoku J Exp Med. 2001;194:55-63. https://doi.org/10.1620/tjem.194.55. 
8. Hyodo I, Eguchi K, Nishina T, Endo H, Tanimizu M, Mikami I, et al. Perceptions and attitudes of clinical oncologists on complementary and alternative medicine: a nationwide survey in Japan. Cancer. 2003;97:2861-8. https://doi.org/10.1002/cncr.11402.

9. Fujiwara K, Imanishi J, Watanabe S, Ozasa K, Sakurada K. Changes in attitudes of Japanese doctors toward complementary and alternative medicinecomparison of surveys in 1999 and 2005 in Kyoto. Evid Based Complement Alternat Med. 2011;2011:608921. https://doi.org/10.1093/ecam/nep040.

10. Arai M, Arai A, Izumi S. Postgraduate education of traditional Japanese (Kampo) medicine: a current survey on the training hospitals in Kanagawa prefecture. Tokai J Exp Clin Med. 2014;39:186-92.

11. Arai M, Katai S, Muramatsu S, Namiki T, Hanawa T, Izumi S. Current status of Kampo medicine curricula in all Japanese medical schools. BMC Complement Altern Med. 2012;12:207. https://doi.org/10.1186/1472-6882-12-207.

12. Beijer HJ, de Blaey CJ. Hospitalisations caused by adverse drug reactions (ADR): a meta-analysis of observational studies. Pharm World Sci. 2002;24: 46-54. https://doi.org/10.1023/A:1015570104121.

13. Wolff JL, Starfield B, Anderson G. Prevalence, expenditures, and complications of multiple chronic conditions in the elderly. Arch Intern Med. 2002;162:2269-76. https://doi.org/10.1001/archinte.162.20.2269.

14. Kaufman DW, Kelly JP, Rosenberg L, Anderson TE, Mitchell AA. Recent patterns of medication use in the ambulatory adult population of the United States: the Slone survey. JAMA. 2002;287:337-44. https://doi.org/10. 1001/jama.287.3.337.

15. Fried TR, O'Leary J, Towle V, Goldstein MK, Trentalange M, Martin DK. Health outcomes associated with polypharmacy in community-dwelling older adults: a systematic review. J Am Geriatr Soc. 2014;62:2261-72. https://doi. org/10.1111/jgs.13153.

16. Charlson ME, Pompei $P$, Ales KL, MacKenzie CR. A new method of classifying prognostic comorbidity in longitudinal studies: development and validation. J Chronic Dis. 1987:40:373-83. https://doi.org/10.1016/0021-9681(87)90171-8.

17. The Japan Geriatrics Society. Guidelines for medical treatment and its safety in the elderly (in Japanese). 2015. https://www.jpn-geriat-soc.or.jp/info/ topics/pdf/20170808_01.pdf. Accessed 22 Nov 2017.

18. Calhoun DA, Jones D, Textor S, Goff DC, Murphy TP, Toto RD, et al. Resistant hypertension: diagnosis, evaluation, and treatment. A scientific statement from the American Heart Association professional education Committee of the Council for high blood pressure research. Hypertens. 2008;51:1403-19. https://doi.org/10.1161/HYPERTENSIONAHA.108.189141.

19. Ohkubo T, Obara T, Funahashi J, Kikuya M, Asayama K, Metoki H, et al. Control of blood pressure as measured at home and office, and comparison with physicians' assessment of control among treated hypertensive patients in Japan: first report of the Japan home versus office blood pressure measurement evaluation (J-HOME) study. Hypertens Res. 2004;27:755-63. https://doi.org/10.1291/hypres.27.755.

20. Mori H, Ukai H, Yamamoto H, Saitou S, Hirao K, Yamauchi M, et al. Current status of antihypertensive prescription and associated blood pressure control in Japan. Hypertens Res. 2006;29:143-51. https://doi.org/10.1291/ hypres.29.143.

21. Hollingsworth JM, Wilt TJ. Lower urinary tract symptoms in men. BMJ. 2014; 349:g4474. https://doi.org/10.1136/bmj.g4474.

22. Wood LN, Anger JT. Urinary incontinence in women. BMJ. 2014;349:g4531. https://doi.org/10.1136/bmj.g4531.

23. Juma S. Urinary retention in women. Curr Opin Urol. 2014;24:375-9. https:// doi.org/10.1097/MOU.0000000000000071.

24. Bush TM, Rayburn KS, Holloway SW, Sanchez-Yamamoto DS, Allen BL, Lam T, et al. Adverse interactions between herbal and dietary substances and prescription medications: a clinical survey. Altern Ther Health Med. 2007;13:30-5.

25. Dergal JM, Gold JL, Laxer DA, Lee MS, Binns MA, Lanctôt KL, et al. Potential interactions between herbal medicines and conventional drug therapies used by older adults attending a memory clinic. Drugs Aging. 2002;19:87986. https://doi.org/10.2165/00002512-200219110-00005.

26. Loya AM, González-Stuart A, Rivera JO. Prevalence of polypharmacy, polyherbacy, nutritional supplement use and potential product interactions among older adults living on the United States-Mexico border: a descriptive, questionnaire-based study. Drugs Aging. 2009;26:423-36. https://doi.org/10.2165/00002512-200926050-00006.

27. Djuv A, Nilsen OG, Steinsbekk A. The co-use of conventional drugs and herbs among patients in Norwegian general practice: a cross-sectional study. BMC Complement Altern Med. 2013;13:295. https://doi.org/10.1186/ 1472-6882-13-295
28. Tommelein E, Mehuys E, Petrovic M, Somers A, Colin P, Boussery K. Potentially inappropriate prescribing in community-dwelling older people across Europe: a systematic literature review. Eur J Clin Pharmacol. 2015;71: 1415-27. https://doi.org/10.1007/s00228-015-1954-4.

29. Davidoff AJ, Miller GE, Sarpong EM, Yang E, Brandt N, Fick DM. Prevalence of potentially inappropriate medication use in older adults using the 2012 beers criteria. J Am Geriatr Soc. 2015;63:486-500. https://doi.org/10.1111/jgs.13320.

30. Yamashita H, Tsukayama H, Sugishita C. Popularity of complementary and alternative medicine in Japan: a telephone survey. Complement Ther Med. 2002;10:84-93. https://doi.org/10.1054/ctim.2002.0519.

31. Hori S, Mihaylov I, Vasconcelos JC, McCoubrie M. Patterns of complementary and alternative medicine use amongst outpatients in Tokyo, Japan. BMC Complement Altern Med. 2008;8:14. https://doi.org/10. 1186/1472-6882-8-14

32. Arai YC, Yasui H, Isai H, Kawai T, Nishihara M, Sato J, et al. The review of innovative integration of Kampo medicine and western medicine as personalized medicine at the first multidisciplinary pain center in Japan. EPMA J. 2014;5:10. https://doi.org/10.1186/1878-5085-5-10.

33. Flaherty JH, Takahashi R, Teoh J, Kim Jl, Habib S, Ito M, et al. Use of alternative therapies in older outpatients in the United States and Japan: prevalence, reporting patterns, and perceived effectiveness. J Gerontol A Biol Sci Med Sci. 2001;56:M650-5.

34. A World Health Organization. WHO Monographs on Selected Medicinal Plants - 1. http://apps.who.int/medicinedocs/en/d/Js2200e/20.html. Accessed 14 Apr 2018

35. Ekor $M$. The growing use of herbal medicines: issues relating to adverse reactions and challenges in monitoring safety. Front Pharmacol. 2014;4:177. https://doi.org/10.3389/fphar.2013.00177.

36. Takayama S, Iwasaki K. Systematic review of traditional Chinese medicine for geriatrics. Geriatr Gerontol Int. 2017;17:679-88. https://doi.org/10.1111/ggi.12803.

37. Steinman MA, Landefeld CS, Rosenthal GE, Berthenthal D, Sen S, Kaboli PJ. Polypharmacy and prescribing quality in older people. J Am Geriatr Soc 2006;54:1516-23. https://doi.org/10.1111/j.1532-5415.2006.00889.x

\section{Ready to submit your research? Choose BMC and benefit from:}

- fast, convenient online submission

- thorough peer review by experienced researchers in your field

- rapid publication on acceptance

- support for research data, including large and complex data types

- gold Open Access which fosters wider collaboration and increased citations

- maximum visibility for your research: over $100 \mathrm{M}$ website views per year

At BMC, research is always in progress.

Learn more biomedcentral.com/submissions 